\title{
Development of Training Equipment for Upper Limb Muscle Rehabilitation
}

\author{
Fengzhi Dai, Yuanli Yue, Qijia Kang, Bo Liu, Wenlong Han, Hongtao Zhang \\ College of Electronic Information and Automation, Tianjin University of Science and Technology \\ 1038 Dagunanlu Road, Hexi District, Tianjin, 300222, China \\ E-mail:daifz@tust.edu.cn \\ www.tust.edu.cn
}

\begin{abstract}
For patient of limb paralysis, the upper limb rehabilitation equipment is important for them to train the muscles. The equipment should be secure, useful and comfortable. They are ensured by the design of mechanical structure and control system. The single joint motion and combinatorial motion are introduced to imitate the function of human upper limb. The upper limb strength can be recovered efficiently and the muscle contraction can also be well preserved. The vibration module and wireless transceiver improve the training effect.
\end{abstract}

Keywords: upper limb, muscle rehabilitation, training equipment, muscle contraction

\section{Introduction}

People pay more attention to the improvement of life quality. However, some diseases such as apoplectic hemiplegia seriously threaten the human health.

The symptom of apoplectic hemiplegia is mainly expressed by the patient's physical activity, which is that the motion of limbs can't be accurately controlled by the brain. The clinical manifestations of this kind of diseases are paralysis or half paralyzed.

It will cause the muscle belly shortening within a short time, and then it will harden the soft tissues that are near to the muscles and joints, thus the function of muscle contraction is gradually lost and lead to muscle atrophy, which is called disuse atrophy.

It is important to develop the training equipment that can help patients for limb muscle rehabilitation ${ }^{1}$.

Rehabilitation training can effectively alleviate the exacerbation of paralysis. After insisting on the arm rehabilitation exercise, the muscle cross-sectional area can be increased, so as to enlarge the muscle fibers. Then the strength and muscular endurance on the shoulder are enhanced so as to prevent muscle atrophy around the shoulder joint ${ }^{2}$. Muscles exercise of upper limb can also speed up the blood flow, and indirectly enhances the cardiovascular function.

Upper limb rehabilitation equipment is a kind of modern device that can help paralyzed patients avoid to lose the function of upper limbs and help rehabilitation of upper limb muscle. But this kind of auxiliary recovery equipment research is still in its infancy.

There are seldom finished products on sale for family usage and most of them have limitations. In fact, there are lots of difficulties that have not been overcame in technical and functional aspects ${ }^{3,4}$. Key points are mainly expressed as follows:

(1) Safety. It is the primary premise. Safety should be considered firstly in the rehabilitation process. The limitation of human upper limb movement should be considered so as to avoid harmless to the patient.

(2) The man-machine interface. Complete relaxation during the recovery process will enhance the recovery effect. It requires that the equipment should be easy use. 
(3) Portability. If the training equipment is too large and heavy, it is not suitable to be used at home. The recovery equipment should be small and easy to move.

(4) Training time. The equipment should work for a long time so as to ensure the time of rehabilitation.

Many experts have developed the upper limb rehabilitation equipment ${ }^{5}$. Robotherapist is the training robot with six degrees of freedom, which was designed by the Takehitokiuchi team from Osaka University, Japan. The robot uses the external traction structure, and it can feed back the training information by sampling the hand position. Patients can also get the information during training process through the screen with the virtual reality technology.

Kinsman company developed the equipment with pulley winded structure to motive patient upper limb achieving rehabilitation training ${ }^{6}$. By the simple structure, patients can be easily trained at home. But it only provides seldom types of exercise for training.

\section{Mechanical structure}

Upper limb auxiliary recovery equipment should bear the pressure of human arm and pulling force. The high strength aluminum plate and organic glass are used. The organic glass plate is bent into a radian that is fit to the upper limb. The model of designed equipment is shown in Fig. 1.

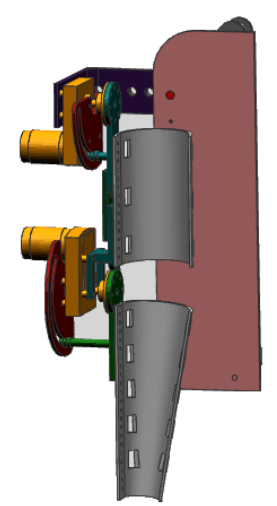

Fig.1. The model of the equipment

The material is $6 \mathrm{~mm}$ thick aluminum plate. The density of aluminum plate is $\rho=2700\left(\mathrm{~kg} / \mathrm{m}^{3}\right)$. If the weight of person is $75 \mathrm{~kg}$, the weight of the person's upper limb is about $3.5 \mathrm{~kg}^{7}$. Through the data calculation and the finite element analysis, the $6 \mathrm{~mm}$ thick aluminum plate can support the weight of the human upper limb and meet the required strength requirements.

\section{Rehabilitation equipment}

The equipment is shown in Fig.2.

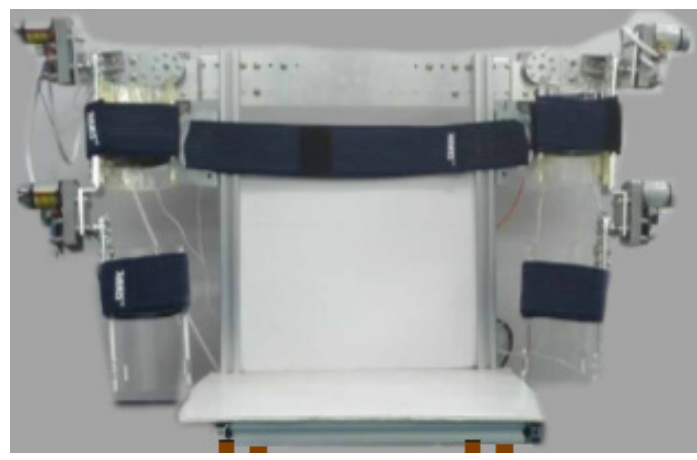

Fig 2. The developed equipment

\subsection{Control system}

The chip for master control is the freescale 32-bit Kinetis (ARM Cortex the M4) micro controller. It has the advantages of high performance, low cost and low power consumption.

The control system stores several sets of upper limb muscle rehabilitation actions. Doctors and family members of patients choose different training patterns for the patients. The functions of control system are:

(1) Six PWM signals are used to control the high torque steering motors.

(2) Alarm module is used to monitor the limitation of motion angles.

(3) LCD displays the current mode and state of training in real time.

(4) Small keyboard and remote controller are used to switch the different training modes.

\subsection{Mechanical restriction}

The upper limb rehabilitation equipment is designed to help patients recover on a safe and comfortable way. As a result, the extreme dimension is necessary to be set for the equipment so as to avoid damage during training.

According to the human upper limb physiological data, the maximum angle of lifting arm upward is $170^{\circ}$ and extending arm backward is $40^{\circ}$. The range of swing arms left and right is $0^{\circ}$ to $70^{\circ}$. 
In order to prevent accident that do harm to the patient during the recovery process, the security range that should not exceed the limit angle is set as follows: the range of lifting arm upward is $0^{\circ} \sim 130^{\circ}$ and extending arm backward is $0^{\circ} \sim 10^{\circ}$, the range of swing arms left and right is $0^{\circ} \sim 30^{\circ}$.

\subsection{Alarm function}

In the recovery process, if the movement of the equipment will exceed the limited range, the angular monitor will detect it and at the same time, it will send the feedback signal to the main control chip, and then it triggers the alarm to prohibit the action immediately.

It makes the equipment working in a safe range and prevent damage to patients. In a word, the mode of automatic alarm and reset has a protective effect on patients and enhances safety performance ${ }^{8}$.

\subsection{Other functions}

The liquid crystal display is used to show the current training patterns and the rehabilitation training time.

It is also equipped with the vibration module. In the recovery process, the vibrating plate is attached to the patient's arm. This function stimulates the patient's muscles, blood circulation and peripheral nerve.

The wireless transceiver module can monitor and transmit the date of training process ${ }^{9}$. By the module, the doctor can monitor the patient's training state remotely, and change the training program in real time. The computer terminal can summarize and analyze the recovery information, and record the training data, which helps the doctors to analyze and improve the training modes.

\section{Medical foundation of upper lamb training}

The movement of the shoulder joint is accomplished with the movement of the arm. According to the theory of the human anatomy, when the body stands on the ground vertically, the axis that is perpendicular to the ground and through the body is called the vertical axis, the axis that through the body of the front and rear and is perpendicular to vertical axis is called the sagittal axis, and the axis that through the body and is perpendicular to the above two axes is the coronal axis.

Based on these three axes, the shoulder joint motion is defined into the following three types ${ }^{10}$. And some motion types of the equipment are shown in Fig.3.

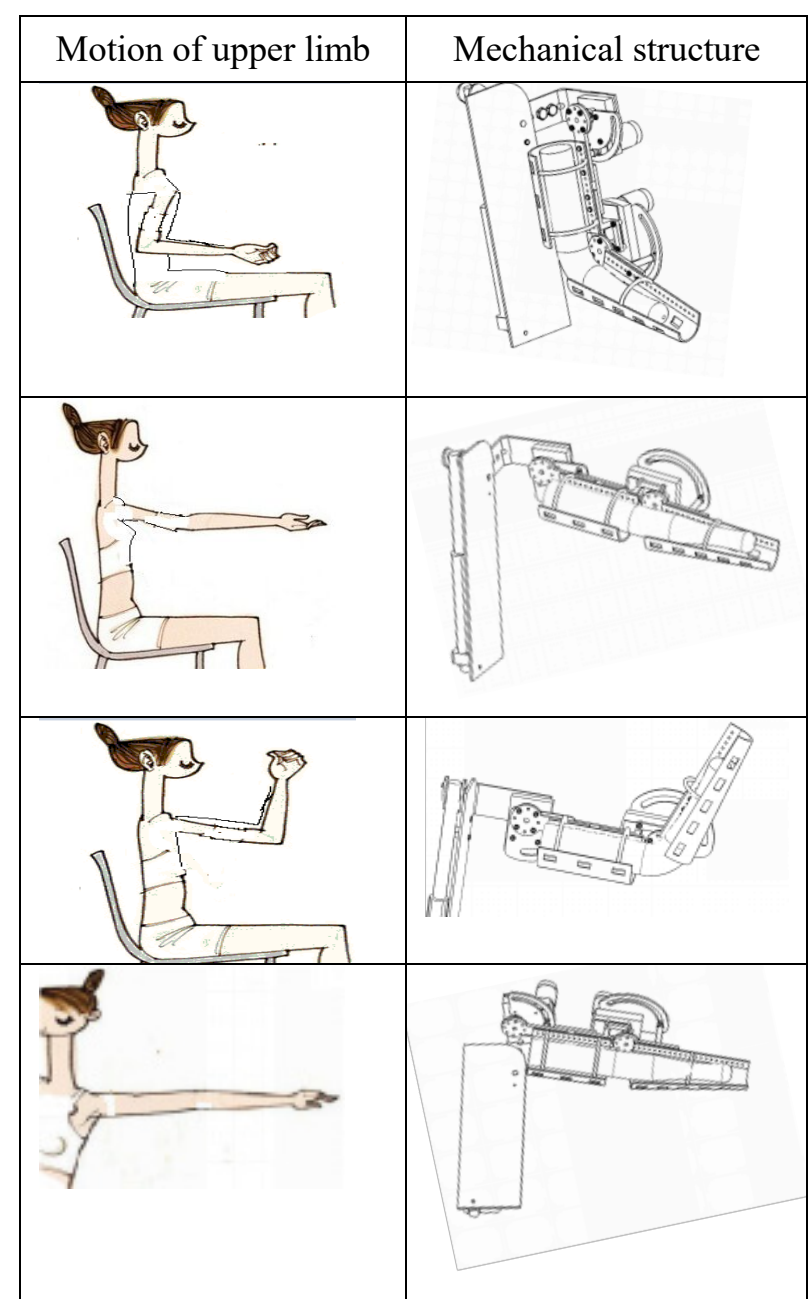

Fig.3. Some types of training

(1) Flexion and extension: Arm moves around the coronary axis in the sagittal plane. Forward motion is flexion and backward is extension.

(2) Outreach and adduction: Arm moves around the sagittal axis in the coronal plane. Moving away from the trunk is outreach and near to the trunk is adduction.

(3) Extorsion and intortion: Arm moves around the vertical axis in the horizontal plane. Moving away from the trunk is extorsion and near to the trunk is intortion.

Also, the upper limb movement is divided into two categories in accordance with the degree of freedom of the movement.

(1) Single joint movement: it is only the training for a single joint, such as the elbow bend and stretch. It is always used at the early stages of training process, or to improve the strength of a particular muscle group. It can strengthen the impression of the nervous system to 
specific actions and promote the rapid recovery of neural function.

(2) Combinatorial movement: it is the most popular way in rehabilitation exercise. Patient's muscle groups must be trained through multiple joints movement. Combinatorial movement can train the coordination of muscle and the control of multiple muscle groups. Combinatorial movement is mainly executed by the way to finish a specific task, such as to carry a cup and drink water.

\section{Conclusion}

The rehabilitation equipment is designed based on the analysis of the medical knowledge, mechanical structure, the construction of the circuit system, combined with the test data.

Synthetically considering the upper limb muscle rehabilitation equipment should be secure, useful and comfortable, the equipment must be:

(1) From a security perspective, the extreme dimension of motion for human upper limb is limited. And the alarm module is used to monitor the system.

(2) From the point to achieve the best recovery effect, different action modes are set to train different muscles.

(3) From the humanistic perspective, the LCD shows the currently executed motion patterns, and the patterns can be adjusted and changed according to the need. Also, the mechanical structure can be adjusted slightly to adapt to different patients.

The result of test and the effect of training shows that the developed upper limbs muscle rehabilitation training equipment is reasonable.

\section{Acknowledgements}

The research is partly supported by the Research Fund for the Doctoral Program of Higher Education of China (20131208110005). It is also supported by the Science \& Technology Fund Planning Project of Tianjin Higher Schools (20140710-1400020005).

\section{References}

1. Yonglian Zhu, Ruihua Wang, Dinghua Fang, Neuro rehabilitation, Beijing, People's Military Medical Publisher, 2001.

2. Ming Yang, Research on rehabilitation training device of elbow join, WuHan, Huazhong University of Science and Technology, 2008

3. Yuchuan $\mathrm{Hu}$, Linhong Ji, Discussion on the design of rehabilitation training robot for upper limb rehabilitation from the medical point of view, Chinese Journal of Clinical Rehabilitation, 8(34): 7754-7756, 2004.

4. Hesheng Wang, Yunhui Liu, Dongxiang Zhou, Adaptive Visual Servoing Using Point and Line Features With an Uncalibrated Eye-in-Hand Camera, IEEE Transactions on Robotics, 24(4), AUGUST 2008.

5. Feng Cai, Design and research of shoulder joint rehabilitation training robot, Dalian, Dalian Jiaotong University, 2012.

6. Dequan Guo, Hui Ju, Yuqin Yao, Research of Manipulator Motion Planning Algorithm Based on Vision, 2009 Sixth International Conference on Fuzzy Systems and Knowledge Discovery, 2009.

7. GB1000-88. Human dimensions of Chinese adults, Beijing: China Standards Press, 1988

8. J Zhang, XM Jiang, Y Chen, Effects of F-wave on early rehabilitation of stroke patients with hemiplegia, Journal of Apoplexy \& Nervous Diseases, 25(1):90-92, 2008.

9. B. Widrow, S. D. Steams, Adaptive Signal Processing, Prentice Hall, Englewood, NJ, 1995.

10. Qiaoyun $\mathrm{Li}$, Rehabilitation nursing of patients with hemiplegia shoulder, Journal of Changzhi Medical College, 3: 14, 2000. 